\title{
QUALITY EVALUATION OF MIXING FODDER BY MOBILE COMBINED UNITS
}

\author{
Vasyl Khmelovskyi, Svitlana Rogach, Oksana Tonkha, Yuriy Rosamaha \\ National University of Life and Environmental Sciences of Ukraine, Ukraine \\ hmelvas@ukr.net,rogach_sm@ukr.net, oksana16095@gmail.com,rosamaha.vchenyij@ukr.net
}

\begin{abstract}
The results of the study of the fodder preparation quality by the combined feeding unit that provides simultaneous grinding and mixing the components with the following dosed distribution to the cattle are given. The method of assessing uniformity of feed mixing for the distribution of moisture was tested. Hypothesis of the method for assessing uniformity of fodder mixtures and without making any selection control component is based on the redistribution of moisture feed components to each other in the process of mixing. The production tested method of assessing of the mixture quality was conducted during the preparation of feed for cattle by a mobile fodder preparation combined unit, which provided simultaneous grinding and mixing of the components with the following dosed distribution of them into the troughs or on the feed tables. Dosage of the starting components, when loading them into the hopper, was performed by using of the weighted mobile fodder preparation combined unit. Moisture content of feed ingredients and feed mixtures was determined by the moisture tester VLK-01, a grain moisture tester also can be used (similar Wile-55, Finland). Indicators of the evaluation of the mixing quality according to the method of moisture distribution were compared with the results of no uniformity mixing, defined by the standard method, which involves the addition of a control component (sugar beet seeds) in the amount of $1 \%$ relative to the weight of the feed mixture. After downloading of the latest component into the feed hopper unit, the minimum acceptable requirements of livestock uniform mixing $(85 \%)$ is provided by stirring for 6-8 minutes. Indicators of the assessment of mixing by the method of moisture distribution and control component practically coincided during the time of feed processing.
\end{abstract}

Keywords: feed mixture, humidity, fodder unit, feeding cows, TMR-technology.

\section{Introduction}

The basis of intensive livestock is known to be full feeding, which provides sufficient amount of feed production, minimizing their losses during harvesting and storage, as well as proper and quality preparation for feeding. The major factors of feeding are [1]: a complete set of essential nutrients, timely and optimally coordinated quantitatively flow to animals.

For sufficient meeting the needs of cattle feed rations it should be balanced about 20 normalized indicators for cattle and 50-80 indicators - for pigs and poultry [2]. And the number of the monitored indicators increases with increasing the intensification level of livestock.

The results of the research and manufacture experience show that the maximum technological efficiency (livestock products) feed resources only in processed form as a part of complete balanced mixtures provide maximum benefit. It is established, for example [3], when the diets of cattle(ruminants) lack 20-25\% digestible protein, the yield is reduced by $30-35 \%$. This feed unit costs increased by $30-40 \%$, and the cost of manufactured products increased by 1.5 times. Costs for processing feed in the process of preparation for feeding is 3-4 times less than the cost additionally obtained due to animal products [4]. However, in the future, we must specifically focus on how to get more milk from each unit of feed rather than simply on how to get more milk from each cow.

Thus, the increasing of the livestock efficiency is impossible without proper preparation of fodder for feeding [5]. This process may include various manufacturing operations, among which the most important and mandatory are crushing of components and preparation of balanced mixtures [6].

During the process of fodder preparation the quality of grinding products is determined by the average particle size and the uniformity of feed fractional composition; the mixing quality criterion is the uniformity of distribution of feed components together in the composition of the feed mixture. The latter index is important when preparing the feed mixtures not for individual cattle, but for the entire process groups [7].

Traditionally, to assess the uniformity of mixing the control component is used (calibrated seed of beets, corn, millet, etc.), which is added to the feed mixture in small quantities [8]. This method takes more time and significant labor costs for its implementation and sometimes requires special laboratory equipment. In case of production inspection the control components must be safe for further feeding of fodder mixture for cattle. It should also be noted that in cases of simultaneous grinding and mixing of feeds the variant with the use of control components of organic origin is much more complicated, 
because after their crushing it is completely impossible to distinguish them from the obtained mixtures.

As it was mentioned above, the search and development of new methods to assess the quality of preparation of complete, balanced feed mixtures are very important and have a scientific and practical significance.

\section{Materials and methods}

There are different ways to assess the quality of mixing powdered feed components. Thus, in [9] it is described how to assess the uniformity of mixing in preparation of animal feed additive with liquid fat. The coefficient of uniformity is determined by digital image processing. With simultaneous grinding and mixing of feed ingredients for the control component [10] iron powder was used with the particle size $0.2-1 \mathrm{~mm}$, it was used allowing to separate it from the samples of the prepared mixtures by a magnet.

There is a possibility of determining the distribution uniformity of forages when tested the shredder-mixtures for such index as moisture basis [11], but this option has virtually no assessment of the production test.

Known methods make it possible to use the minimum permissible sample for concentration of the control component to figure the uniformity coefficient of mixture [12]. However, the ways and methods of assessing the quality of the mixing process, in particular the uniformity of the fodder mixtures, require further research and improvement for simplification of the methods and reduction of the complexity of their implementation [13].

The purpose of the work. Testing the method for assessing the quality (uniformity) of mixing through redistribution of moisture in fodder mixtures for cattle [14].

The results of the research. Hypothesis of the method for assessing uniformity of fodder mixtures and without making any selection control component based on the redistribution of moisture feed components to each other in the process of mixing.

According to the given composition of feed intake (normalized for dose and source components that are part of it) before preparing of the feed mixture the moisture content of each component till download into the hopper of the mixer should be determined. Then you calculate the expected (theoretical) mixture humidity $W_{s t}$, an average index of the known formula (1):

$$
W_{\mathrm{st}}=\frac{m_{1} W_{1}+m_{2} W_{2}+\ldots+m_{n} W_{n}}{m_{1}+m_{2}+\ldots+m_{n}},
$$

where $m_{1}, m_{2}, m_{n}$ - mass of starting components that make up the feed ration, $\mathrm{kg}$;

$W_{1}, W_{2}, W_{n}$ - humidity of the appropriate feed ingredients, $\%$.

During the preparation of the feed mixture the samples are taken and their actual humidity is determined $W_{f}$. Comparison of the actual and theoretical moisture allows to estimate the distribution of moisture in the whole volume of the prepared mixtures, hence the uniformity of mixing feed components. The degree of non-uniformity mixing or deviation from the expected value $\delta$ is (2):

$$
\delta=\frac{W_{s t}-W_{f}}{W_{s t}} 100 \%
$$

For accurate assessment of mixing uniformity for the moisture distribution the samples should be selected from different zones of the mixer volume of the batch actions or at the regular intervals at the output of the streaming (continuous) mixer. Numbers of samples are taken depending on the structural composition of feed rations from operating zoo technical requirements as to the uniformity mixtures and necessary probability of the result. Referring to the results of the research and industrial practice tests of feed mixtures, the number of samples can be recommended between 10 and 120. In [7] it is observed that in the wet preparation of mixtures with the inclusion of the stem and juicy fodder 2040 samples provide probable results 0.95 . 
The mass of the sample $M_{p}$ is recommended [5] to take with the conditions for achieving the required accuracy of assessment. For options with a control component $M_{p}$ is given by:

$$
M_{p}=10 \quad V_{c} \frac{\varepsilon^{2}}{\alpha^{2}}\left(\frac{\rho_{\kappa \kappa}}{k}-\rho_{\kappa c}\right),
$$

where $V_{c}$-average volume of a particle of the control component $\mathrm{cm}^{3}$;

$\rho_{\kappa \kappa}-$ density of the control component; $\mathrm{kg} \cdot \mathrm{cm}^{-3}$;

$\rho_{\kappa c}-$ density of the feed mixture, $\mathrm{kg} \cdot \mathrm{cm}^{-3}$;

$\alpha$ - given significance level (probability for $\varepsilon=0.95, \alpha=1.96$ );

$k$-weight of the control component in the mixture, $k=0.01$.

In practice in testing of agricultural machinery, when the quality of the mass mixtures is determined, the samples are taken for fodder mixtures 5 grams, wet mixtures for pigs and dry mixtures for cattle 100 grams and for wet mixtures for cattle 300-500 grams.

In a simplified form (without determining the moisture of the starting components and the expected mixture humidity) of the assessment of mixture uniformity the moisture of the prepared mixtures is accepted as a controlled setting.

Then moisture uniformity is determined in the samples taken from the entire volume of mixtures.

The production tested method of assessing the mixtures quality was conducted during the preparation of feed for cattle (Fig. 1) by a mobile fodder preparation combined unit (MFPCU), which provided simultaneous grinding and mixing of the components with the following dosed distribution of them into the troughs or on the feed tables.

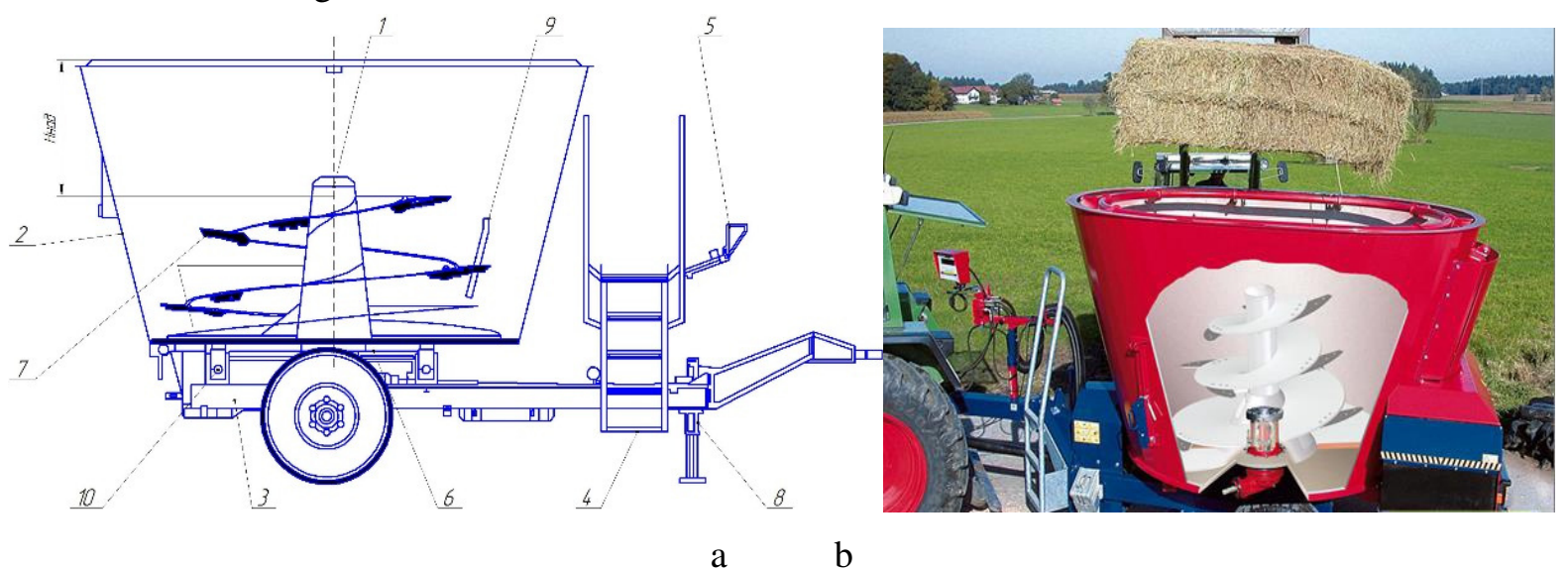

Fig.1. Scheme (a) andgeneralview (b) MFPCU: 1 - screw; 2 - bunker; 3 - frame; 4 - ladder;

5 - display of the weighing system; 6 - reducer; 7 - knife; 8 - paw supports; 9 against cuts; 10 - sensor weighing system

Dosage of the starting components, when loading them into the hopper, was performed by using the weighted unit MFPCU.

Moisture content of feed ingredients and feed mixtures was determined by the moisture tester VLK-01, a grain moisture tester also can be used (similar Wile-55, Finland).

In the process of preparing the feed mixture in the MFPCU, when loading the fodder components according to the recipe, mixing takes place on the principle of mixing conditionally two fodder components.

This assertion is based on the principle of MFPCU.

After loading of the first component into the feed hopper, the last unit moves to the next and at the same time the loaded component is crushed.

Then another component is added to the tank, where further grinding with simultaneous mixing of the downloaded components to each other takes place, thus forming mixtures with other physical and mechanical properties that are different from the original. 
To these previous mixtures as a conventional single component the next component from the storage is added, etc.

Indicators of the evaluation of the mixing quality according to the method of moisture distribution were compared with the results of no uniformity mixing, defined by the standard method, which involves the addition of a control component (sugar beet seeds) in the amount of $1 \%$ relative to the weight of the feed mixture.

No uniformity mixture in the version with a control component coefficient of variation $v$ is:

$$
v=\frac{100}{\bar{X}} \sqrt{\frac{\sum\left(X_{i}-\bar{X}\right)^{2}}{n-1}},
$$

where $X_{i}$ - concentration of the control component in samples, grams;

$$
\begin{aligned}
& \bar{X}=\frac{\sum_{1}^{n} X_{i}}{n} \text {-arithmetic average deviation of the component concentration; } n \text { - number of } \\
& \text { analyzed samples. }
\end{aligned}
$$

The degree of the mixture uniformity $\delta$ thus is determined by the expression: $\delta=100-v$.

\section{Results and discussion}

The studies show that the process occurs very rapidly (Fig. 2): after downloading of the latest component into the feed hopper unit, the minimum acceptable requirements of livestock feed uniform mixing $(85 \%)$ is provided by stirring for 6-8 minutes.

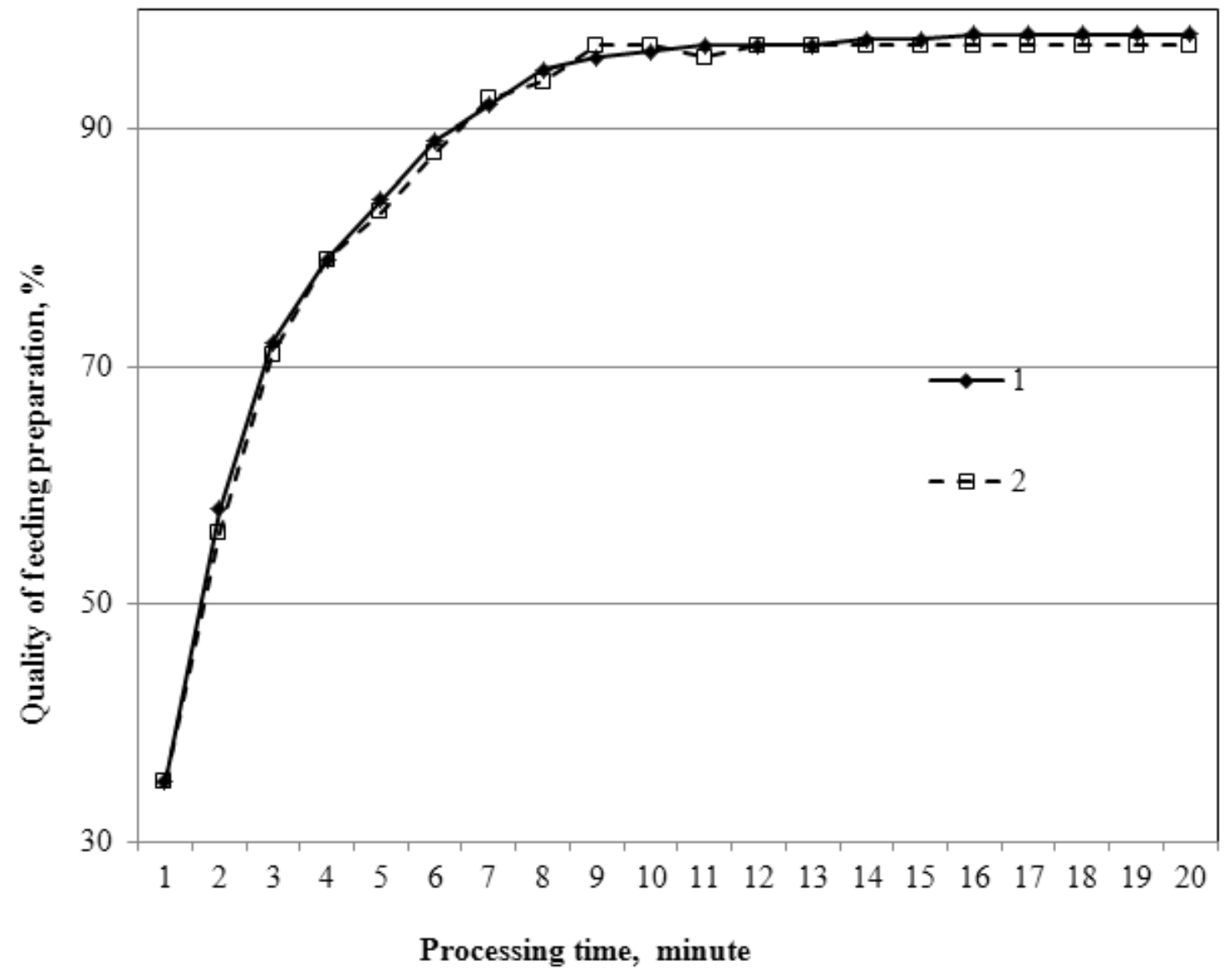

Fig. 2. Dependence of quality of feeding preparation on processing time (after downloading of latest component in bunker MFPCU): 1 - uniformity index $\delta$ was determined by the method of moisturesredistribution; $2-\delta$ defined by the control component 
The indicators of assessment of mixing by the method of moisture distribution and control component practically coincided during the time of feed processing.

All known methods for assessing the quality of mixing, pre-prepared feed components, have some inconvenience when used in production conditions. For example, the homogeneity assessment of mixing mixed fodders with the use of liquid fat additive requires the use of special digital equipment that determines the uniformity of the mixing ratio. Such a technique can be used in preparation of similar types of fodder mixtures. In case of simultaneous shredding and mixing of the ingredients of mixed fodder, for checking the qualitative parameters of the mixture, cast iron powder is used as a control component with a particle size of $0.2-1 \mathrm{~mm}$. The disadvantage of this method is the incomplete allocation of samples from the cooked mixture using a magnet. Samplings of the feed mixture have to be disposed of. Other valuation methods need a lot of time.

The hypothesis of the method for assessing the uniformity of mixing the feed mixture, without introducing and isolating any control component, is based on the redistribution of the moisture of the feed components between themselves in the process of mixing them. The method makes it possible to control the quality indices of the feed components immediately before cooking the feed mixture.First of all, it is necessary to determine the humidity of each component. Based on the results obtained, we calculate the moisture content of the mixture as a weighted average. The simplicity and efficiency of the method is that, in the process of preparing the feed mixture, samples of the mixture are close in weight and volume and determine their actual humidity. Comparison of the actual and estimated humidity allows us to evaluate the uniformity of the mixing of feed components by analyzing the patterns of moisture distribution in the entire volume of the prepared mixture.

Sampling should be carried out in different locations (zones) of the mixer hopper, which allows achieving a high degree of uniformity of mixing. Also, in the version without preliminary determination of the moisture content of the initial components and the calculated moisture of the mixture, the assessment of the uniformity of mixing is carried out according to the steady (approximate) indicators of humidity of the prepared feed mixture.

The proposed method for assessing the uniformity of mixing, by redistributing moisture in feed mixes, can be implemented in mixers as an automatic option.

\section{Conclusions}

1. The testing method for assessing the quality of mixing by redistribution of moisture in feed mixtures for cattle showed the complete adequacy of the results compared with the traditional method of using a control component. This first version is marked by simplicity of implementation, provides the ability to determine quickly the uniformity of mixing and can be used in cases of simultaneous grinding and mixing of feed.

2. The minimum adequate mixing time after the last component in the tank MFPCU is determined.

\section{Acknowledgements}

This work was supported by a grant of the Ministry of Science and Education of Ukraine, contract _110/495/01.01.2016, Project code: UA 1649501 01, NULES Project title - "Experimental researches of parameters and modes of functioning of system of maintenance of agricultural machinery", phase 6: Experimental researches of parameters and modes of functioning of system of operational safety for agricultural aggregates.

\section{References}

[1] Savinyh P., Sychugov Y., Kazakov V., Ivanovs S. Development and theoretical studies of grain cleaningmachine for fractional technology of flattening foragegrain. Proceedings of 17 th International Scientific Conference "Engineering for rural development". Jelgava, Latvia, May 23-25, 2018, Latvia University of Agriculture. Faculty of Engineering. Vol. 17, pp. 124-130.

[2] Pavel Kic. Influence of housing technology on dust level in cowsheds. Proceedings of 17th International Scientific Conference "Engineering for rural development". Jelgava, Latvia, May 23-25, 2018, Latvia University of Agriculture. Faculty of Engineering. Vol. 17, pp. 24-27. 
[3] Butkus R., Vasiliauskas G. Occupational risk factors in small stockbreeding farms including Lithuanian conditions. Proceedings of 17th International Scientific Conference "Engineering for rural development”. Jelgava, Latvia, May 23-25, 2018, Latvia University of Agriculture. Faculty of Engineering. Vol. 17, pp. 1427-1433.

[4] Hassen H.B., Elaoud A., Trabelsi I. Influence of the magnetic fields on some characteristics of raw milk. International Journal of Advance Industrial Engineering, 2017. Vol.5, No. 4, pp. 200204.

[5] Chamberland, J.; Lessard, M.H.; Doyen, A. A sequencing approach targeting the 16S rRNA gene unravels the biofilm composition of spiral-wound membranes used in the dairy industry. Dairy science \& technology Vol: 6, 2017. pp. 827-843.

[6] Alessandro Bonanno, Carlo Russo and Luisa Menapace, Market power and bargaining in agrifood markets: A review of emerging topics and tools, Agribusiness, 34, 1, 2017, pp. 6-23.

[7] Atanov I.V., Kapustin I.V., Grinchenko V.A., Gritsai D.I., Kapustina E.I. Improving efficiency of feeding cattle. Research Journal of Pharmaceutical, Biological and Chemical Sciences. 2016. T. 7. № 4. pp. 1927-1932.

[8] Dolejš J., Mašata O., Toufar O. Elimination of dust production from stables for dairy cows, Czech J. Anim. Sci., 51, 2006 (7), pp. 305-310.

[9] Pascuzzi S., Santoro F. Analysis of the almond harvesting and hulling mechanization process: $\begin{array}{lllllll}\text { A case study. Agriculture (Switzerland), } & 7(12), & 2017, & 100 & \text { p. }\end{array}$ http://dx.doi.org/10.3390/agriculture7120100.

[10] Pascuzzi S. A multibody approach applied to the study of driver injuries due to a narrow-track wheeled tractor rollover. J. Agr. Eng., vol. 46, 2015, pp. 105-114. http://dx.doi.org/10.4081/jae.2015.466.

[11] Valentina Becciolini, Maria Paola Ponzetta. Inferring behaviour of grazing livestock: opportunities from GPS telemetry and activity sensors applied to animal husbandry. Proceedings of International Scientific Conference "Engineering for Rural Development". Volume 17, 2018, Jelgava, Latvia University of Agriculture, Latvia, pp. 192-198.

[12] Gorobets V.G., Trokhaniak V.I., Rogoskii I.L., Titova L.L., Lendiel T.I., Dudnyk A.O., Masiuk M.Yu. The numerical simulation of hydrodynamics and mass transfer processes for ventilating system effective location. INMATEH. Agricultural Engineering. Bucharest. Romania, vol. 56, No 3, 2018, pp. 185-192. Scopus. WoS.

[13] Barvicki J., Gach S., Ivanovs S. Proper utilization of soil structure for crops today and conservation for future generations. Proceedings of 11th International Scientific Conference "Engineering for Rural Development", proceedings, Jelgava, Latvia, May 24-25, 2012, Latvia University of Agriculture. Faculty of Engineering. Vol. 11, pp. 10-15.

[14] Priekulis J., Latvietis J. Economic profitability of milk production. Proceedings of 8th International Scientific Conference "Engineering for rural development". Jelgava, Latvia, May 28-29, 2009, Latvia University of Agriculture. Faculty of Engineering. Vol. 8, pp.81-85. 